\title{
Incomplete midline atrial chamber in a symptom-free adult: An enigma?
}

\author{
Lognathen Balacumaraswami, FRCS, ${ }^{a}$ Siew Yen Ho, PhD, FRCPath, ${ }^{\mathrm{c}}$ Oliver \\ Ormerod, MD, FRCP, ${ }^{\mathrm{b}}$ and Ravi Pillai, FRCS, ${ }^{\text {a }}$ Oxford and London, United \\ Kingdom
}

$\mathrm{T}$ he incidence of atrial septal defect associated with anomalous pulmonary venous drainage is low. This case illustrates the presence of partial anomalous pulmonary venous drainage into a midline atrial chamber with no interatrial communication, a situation not previously described in patients with the usual atrial arrangement.

\section{Clinical Summary}

An incidental heart murmur in a 24-year-old woman led to discovery of an atrial septal defect with an apparent partition on echocardiogram. Transesophageal echocardiography and magnetic resonance imaging showed left pulmonary venous drainage into the left atrium (LA) and right pulmonary venous drainage into a midline atrial chamber that communicated with the right atrium (RA) but was completely partitioned from the LA (Figures 1 and $2, A)$. There were no other cardiac anomalies or visceral malformations. Cardiac catheterization confirmed absence of an interatrial communication and normal pulmonary artery pressure. There was a step up in oxygen saturation in the mid RA (94\%) from the superior vena cava and high RA $(78 \%)$. The main pulmonary artery saturation was $92 \%$, whereas the right upper and lower pulmonary veins accessed through the RA into the midline chamber were $98 \%$ saturated. The left pulmonary venous drainage into the LA was visualized on the levo phase of the left pulmonary angiogram (Figure 2, B).

During the operation, the heart appeared normal externally. Right atriotomy showed a midline atrial chamber, formed by a right-sided incomplete partition and intact left-sided atrial septum, into which opened the orifices of the right upper and lower pulmonary veins. The free margin of the partition was suspended from the septal aspect of the atrioventricular junction and allowed venous return from the right pulmonary veins to flow to the tricuspid valve orifice. The atrial septum and the partition were

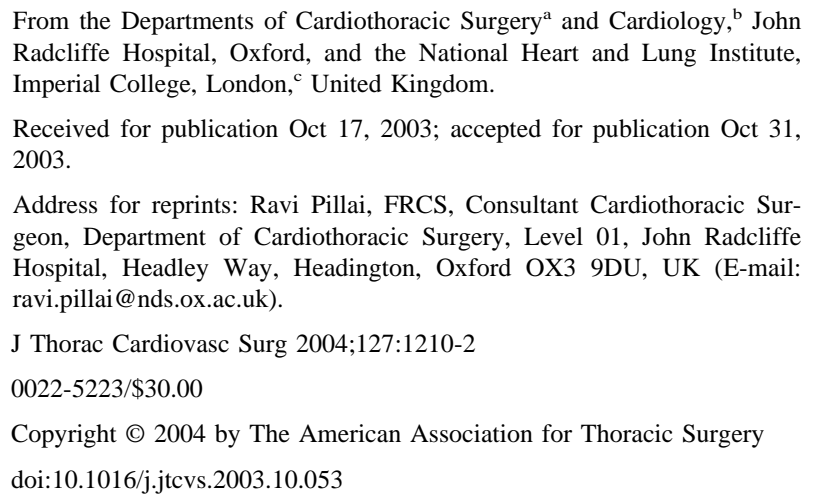

excised, and the resulting common atrial chamber was reseptated with autologous pericardial patch to

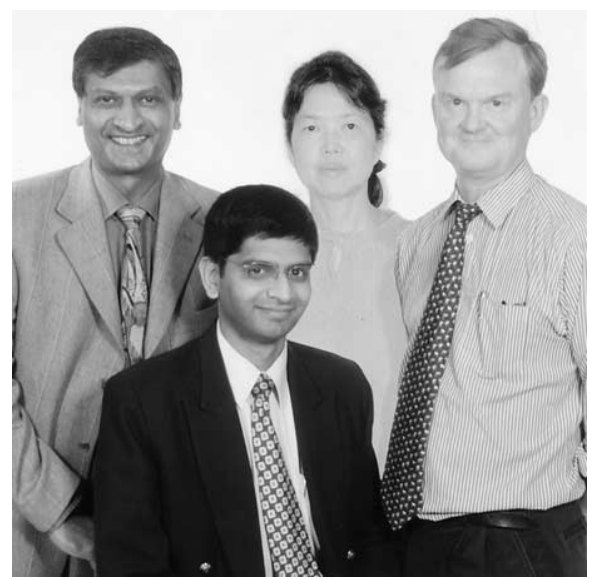

Authors (left to right): Pillai, Balacumaraswami, Ho, and Ormerod allow all pulmonary venous return to be directed into the LA. The eustachian valve and the valve of the coronary sinus were in the anatomically normal position. The patient recovered uneventfully and remains symptom free 7 years after the operation. Postoperative echocardiography confirmed drainage of all pulmonary veins into the LA.

\section{Comment}

The incomplete midline accessory chamber in this case is intriguing, especially because the heart was otherwise normal, with the usual arrangement of the atrial appendages (situs solitus). It is well recognized that hearts with isomeric arrangement of the right atrial appendages (situs ambiguous) not infrequently have the pulmonary veins draining into an atrial pouch in the middle of the roof of the atrial mass. ${ }^{1}$ This is to be anticipated, because most of these hearts have grossly abnormal formation of the atrial septum and totally anomalous pulmonary venous connections according to morphologic criteria. To the best of our knowledge, our case exhibits a most unusual entity, one that has not been described previously. The diagnosis of the midline chamber was made with three imaging modalities. The differential diagnoses considered were partially anomalous pulmonary venous connections and cor triatriatum (also known as subdivided LA). In terms of flow, there is no doubt that the right pulmonary veins drained into the RA instead of the LA. Whether the right veins are also anomalously connected, however, cannot be determined. This is because the embryologic derivation of the intact septum to the left of the midline chamber is open to conjecture. We have considered this structure the atrial septum because in fetal life it must have been patent, at the oval fossa, to allow flow to the left side of the heart for normal development of the chambers on that side.

Alternatively, this case is reminiscent of the case of subdivided LA described by Michaud and colleagues, ${ }^{2}$ in which the left pulmonary veins drained into the distal chamber and the right pulmonary veins drained into the proximal chamber, the so-called type A2 of Thilenius and associates. ${ }^{3}$ The argument against our case being an example of subdivided LA is the configuration of the partition on the right side. The defect in the partition lies toward the atrioventricular junction, in a location comparable to an ostium primum, except that this heart lacks the hallmark of a common 


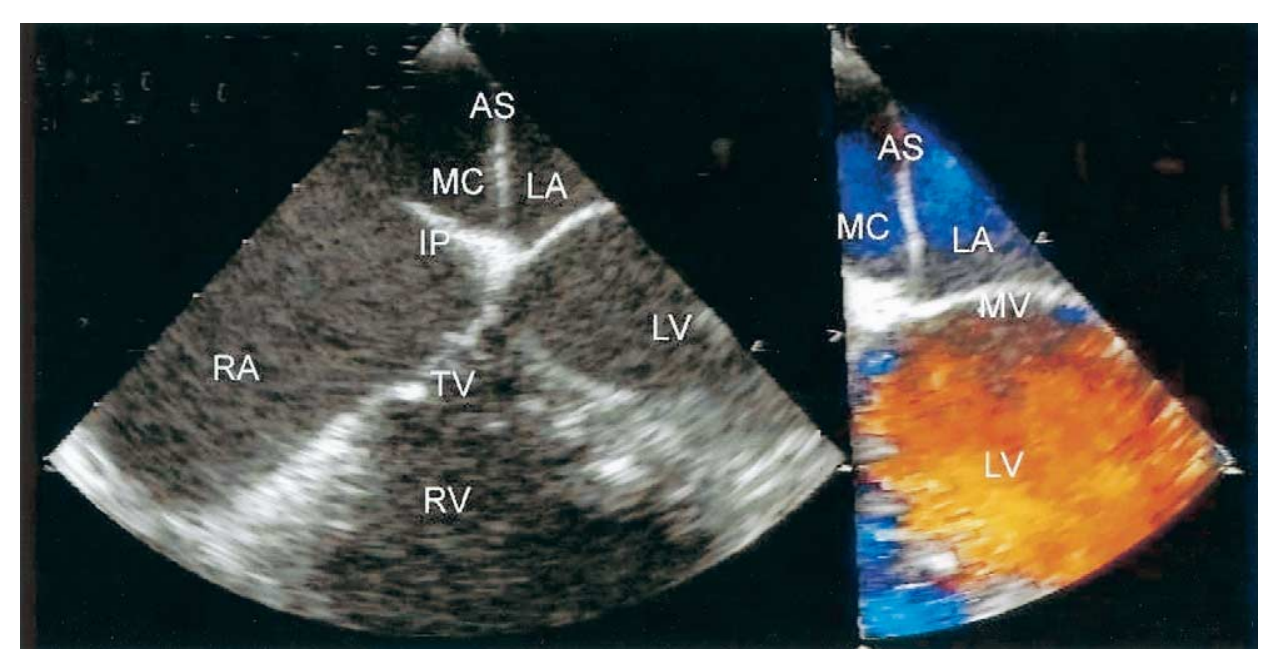

Figure 1. Transesophageal echocardiogram showing midline chamber (MC) between complete atrial septum (AS) and incomplete partition (IP). Atrial septum separates midline chamber from LA. RA, closed tricuspid valve (TV), left ventricle (LV), mitral valve (MV), and right ventricle $(R V)$ are also shown.

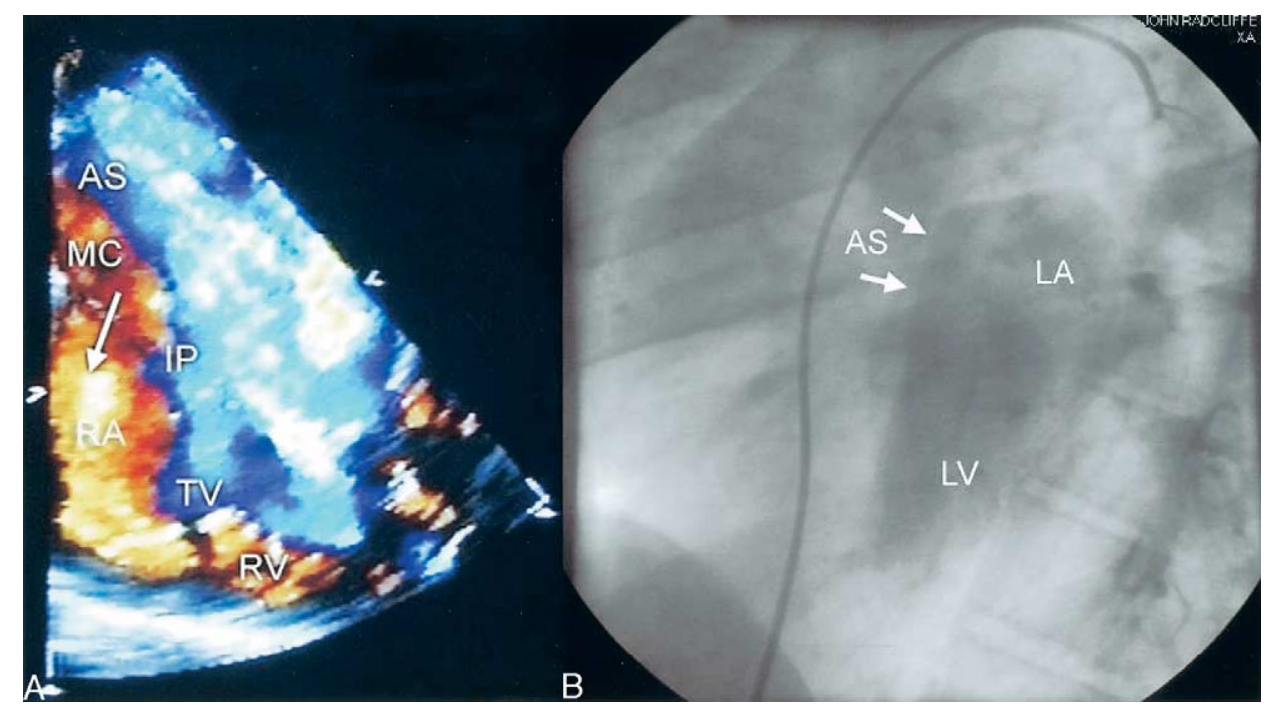

Figure 2. A, Transesophageal echocardiogram shows direction of blood flow (arrow) from midline chamber (MC) bounded by atrial septum (AS) and incomplete partition (IP) into RA and across tricuspid valve (TV) into right ventricle (RV). B, Cardiac catheterization shows occlusive nature (arrows) of atrial septum (AS) on levo phase of left pulmonary angiogram. LA and left ventricle (LV) are seen.

atrioventricular junction that is so characteristic of hearts with atrioventricular septal defects. It has discrete left and right atrioventricular junctions guarded by mitral and tricuspid valves, respectively.

The embryologic derivation of this midline chamber is enigmatic, because the pulmonary venous incorporation into the atrium is closely timed with atrial septal development but the spatiotemporal sequence is an issue of controversy. Currently there are two schools of interpretation of the embryologic origin of the common pulmonary vein, which is the precursor of the definitive lateralized veins. The primary theory suggests the origin of common pulmonary vein within mediastinal tissues that remains distinct from the sinus venosus delineated by the right and the left venous valves. ${ }^{4}$ The alternative theory supports its origin from sinus venosus, with eventual positioning of the common pulmonary vein into the LA. ${ }^{5}$ The septum primum grows into the common atrium and is separated from the left venous valve by the interseptovalvular space. Normally, the muscular septum secundum is formed within the interseptovalvular space and the left venous valve blends with the right side of septum secundum to obliterate this space. In this case, 
however, persistence of interseptovalvular space with the embryonic left venous valve remnant as the incomplete partition may explain the morphogenesis of the midline chamber. Because of marked asymmetry in the timing and sequence of events for pulmonary venous development, a process of differential pulmonary venous segregation, which supports the primary theory, ${ }^{4}$ may explain right pulmonary vein malincorporation into the midline chamber.

Although appreciation of the developmental basis would not have altered the surgical approach, recognition of precise anatomic disposition of this unusual anomaly is essential for effective planning of the appropriate corrective surgical strategy. Awareness of the exact nature of the pulmonary venous drainage is pivotal to the prevention of pulmonary venous obstruction and its ensuing adverse hemodynamic effects.

\section{References}

1. Uemura H, Ho SY, Devine WA, Kilpatrick LL, Anderson RH. Atrial appendages and venoatrial connections in hearts from patients with visceral heterotaxy. Ann Thorac Surg. 1995;60:561-9.

2. Michaud P, Dallaz C, Agé C. A propos d'un nouveau cas de coeur triatrial de l'adulte opéré avec succés. Arch Mal Coeur. 1970;63:291-300.

3. Thilenius OG, Bharati S, Lev M. Subdivided left atrium: an expanded concept of cor triatriatum sinistrum. Am J Cardiol. 1976;37:743-52.

4. Webb S, Kanani M, Anderson RH, Richardson MK, Brown NA. Development of the human pulmonary vein and its incorporation into the morphologically left atrium. Cardiol Young. 2001;11:632-42.

5. Blom NA, Gittenberger-de Groot A, Jongeneel TH, DeRuiter MC, Poelmann RE, Ottenkamp J. Normal development of the pulmonary veins in human embryos and formulation of a morphogenetic concept for sinus venosus defects. Am J Cardiol. 2001;87:305-9.

\section{Completion lobectomy after bilateral lung volume reduction for emphysema: Salvage option or fancy?}

Eugenio Pompeo, MD, Davide Mineo, MD, Patrizio Bollero, MD, Paola Rogliani, MD, Vincenzo Ambrogi, MD, and Tommaso Claudio Mineo, MD, Rome, Italy

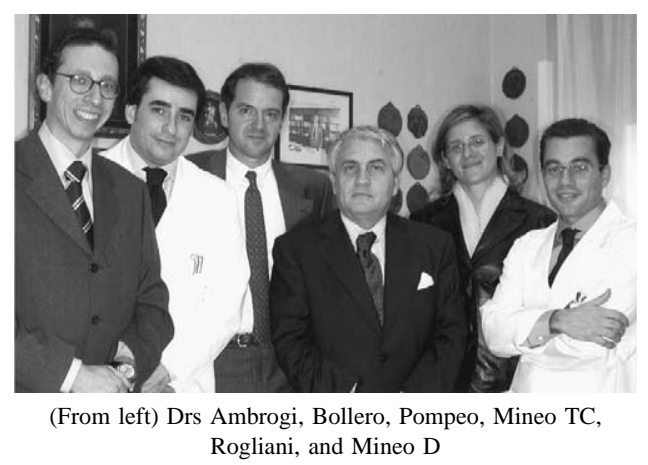

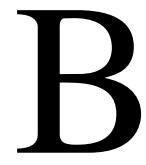

ilateral lung volume reduction (LVR) can significantly improve the functional capacity of selected patients with severe emphysema and an upper-lobe predominance of disease. ${ }^{1,2}$ However, because of its palliative nature, postoperative improvements usually peak within the first 6 months and slowly decline subsequently, eventually returning back to the baseline status in most patients. Little is known, however, as to whether redo $\mathrm{LVR}^{3}$ can be reasonably proposed in selected patients after bilateral LVR.

\footnotetext{
From the Thoracic Surgery and the Multidisciplinary Pulmonary Program, Policlinico University Tor Vergata, Rome, Italy.

This research was supported by Ministero dell'Università e della Ricerca Scientificá e Tecnologica (MURST) Cofinanziamento (COFIN) grants 9906274194-06 and 2001061191-001, Consiglio Nazionale della Ricerca (CNR) CU0100935 2002, and Centro di Eccellenza 2001.

Received for publication Aug 16, 2003; revisions requested Oct 2, 2003; revisions received Nov 3, 2003; accepted for publication Dec 3, 2003.

Address for reprints: Tommaso C. Mineo, MD, Cattedra di Chirurgia Toracica, Università Tor Vergata, Policlinico Tor Vergata, V.le Oxford 81, 00133 Rome, Italy (E-mail: mineo@med.uniroma2.it).

J Thorac Cardiovasc Surg 2004;127:1212-4

$0022-5223 / \$ 30.00$

Copyright $\odot 2004$ by The American Association for Thoracic Surgery

doi:10.1016/j.jtcvs.2003.11.054
}

Herein we describe 4 patients with upper-lobe prevailing emphysema who underwent completion lobectomy (CL) as a redo LVR after a previous bilateral operation. The patients, all men, were operated on between September 2001 and October 2002. All patients gave their written, informed consent. The mean interval between bilateral LVR and CL was 59 months. After the bilateral operation, marked improvements occurred in pulmonary function measures: $+360 \mathrm{~mL}$ in forced expiratory volume in 1 second $\left(\mathrm{FEV}_{1}\right),-1170 \mathrm{~mL}$ in residual volume, $+125 \mathrm{~m}$ in the 6-minute walking test, and $+5.25 \mathrm{~mm} \mathrm{Hg}$ in arterial oxygen tension. Afterward, the $\mathrm{FEV}_{1}$ annual decline averaged $0.23 \pm 0.09 \mathrm{~L}$ at 24 months and $\pm 0.08 \mathrm{~L}$ at 36 months.

$\mathrm{CL}$ was performed when $\mathrm{FEV}_{1}$ returned to baseline values and high-resolution computed tomography showed severe emphysematous lung destruction that was prevailing in 1 upper lobe (Figure 1,1 and 2). Before CL, all patients were receiving maximized medical therapy and underwent a 4-week respiratory rehabilitation program.

CL (3 right upper lobectomies and 1 left upper lobectomy) was performed by video-assisted minithoracotomy ( 2 patients) or lateral thoracotomy ( 2 patients). In 2 instances, isolation of the hilar structures was accomplished in a standard manner. In another 2 patients, the fissure was not dissected free, and the upper lobe tissue was excised through nonbuttressed staple resection (Figure 2). All patients were extubated in the operating room, but 1 required early reintubation and eventually died of respiratory failure. In the remaining 3 patients, there were no major complica- 